\title{
GLOBAL EXPONENTIAL STABILITY AND EXISTENCE \\ OF ANTI-PERIODIC SOLUTIONS \\ TO IMPULSIVE COHEN-GROSSBERG NEURAL NETWORKS ON TIME SCALES
}

\author{
YONGKUN Li — TIANWEI ZHANG
}

\begin{abstract}
By using the method of coincidence degree theory and Lyapunov functions, some new criteria are established for the existence and global exponential stability of anti-periodic solutions to impulsive CohenGrossberg neural networks on time scales. Our results are new even if the time scale $\mathbb{T}=\mathbb{R}$ or $\mathbb{Z}$. Finally, an example is given to illustrate our results.
\end{abstract}

\section{Introduction}

Since Cohen and Grossberg proposed a class of neural networks in 1983 [10], which include Hopfield neural networks [14], shunting neural networks and other neural networks [23], this model has received increasing interest due to its promising potential for applications in classification, parallel computation, associative memory, especially in solving some optimization problems. Such applications rely on the qualitative properties of stability. Thus, the qualitative analysis of these dynamic behaviors is a prerequisite step for the practical design and application of neural networks. Because of the finite speed of switching and

2010 Mathematics Subject Classification. 34K13, 34K20, 34N05, 92B20.

Key words and phrases. Anti-periodic solution, Cohen-Grossberg neural networks, impulse, time scale.

This work is supported by the National Natural Sciences Foundation of People's Republic of China under Grants 10971183 and 11361072. 
transmission of signals in a network, time delays are inevitably present in electronic implementation of neural networks, which may influence the stability of the entire network by creating oscillatory or unstable phenomena. In recent years, the dynamical behaviors of Cohen-Grossberg neural networks with delays have been studied by many researchers (see e.g. [9], [17]-[19], [21], [25]).

In reality, many physical systems undergo abrupt changes at certain moments due to instantaneous perturbations, which lead to impulsive effects. Since the existence of impulses is frequently a source of instability, bifurcation and chaos for neural networks, the impulsive neural networks is an appropriate description of the phenomena of abrupt qualitative dynamical changes of essentially continuous-time systems, see [3], [20], [31], [32] and references therein.

In fact, continuous and discrete systems are very important in implementing and applications. It is well known that the theory of time scales has received a lot of attention which was introduced by Stefan Hilger in order to unify continuous and discrete analysis. Therefore, it is meaningful to study dynamic systems on time scales which can unify differential and difference systems see [20].

Arising from problems in applied sciences, it is well-known that anti-periodic problems of nonlinear differential equations have been extensively studied by many authors during the past twenty years, see [1], [3], [27], [28], [33] and references therein. For example, anti-periodic trigonometric polynomials are important in the study of interpolation problems [11], [12], and anti-periodic wavelets are discussed in [6]. In contrast, however, very few results are available on the existence and exponential stability of anti-periodic solutions for neural networks, while the existence of anti-periodic solutions plays a key role in characterizing the behavior of nonlinear differential equations [8], [22], [29], [30].

To the best of our knowledge, the existence of anti-periodic solutions to impulsive neural networks with time-varying delays has seldom been investigated and remains important and challenging.

Motivated by above, in this paper we are concerned with the following impulsive Cohen-Grossberg neural networks on time scales

$$
\left\{\begin{aligned}
& x_{i}^{\Delta}(t)=-a_{i}\left(x_{i}(t)\right)\left[b_{i}\left(x_{i}(t)\right)-\sum_{j=1}^{n} c_{i j}(t) f_{j}\left(x_{j}(t)\right)\right. \\
&\left.-\sum_{j=1}^{n} d_{i j}(t) g_{j}\left(x_{j}\left(t-\tau_{i j}(t)\right)\right)-e_{i}(t)\right], \quad t \in \mathbb{T}^{+}, t \neq t_{k}, k \in \mathbb{N} \\
& \Delta x_{i}\left(t_{k}\right)=x_{i}\left(t_{k}^{+}\right)-x_{i}\left(t_{k}^{-}\right)=I_{i k}\left(x_{i}\left(t_{k}\right)\right), \quad i=1, \ldots, n
\end{aligned}\right.
$$

where $\mathbb{T}$ is an $\omega / 2$-periodic time scale which has the subspace topology inherited from the standard topology on $\mathbb{R}, \mathbb{T}^{+}=\{t \in \mathbb{T}: t \geq 0\}, \mathbb{N}=\{1,2, \ldots\}$; for each interval $I$ of $\mathbb{R}$, we denote $I_{\mathbb{T}}=I \cap \mathbb{T}, x_{i}\left(t_{k}^{+}\right), x_{i}\left(t_{k}^{-}\right)(i=1, \ldots, n)$ represent the right and left limit of $x_{i}\left(t_{k}\right)$ in the sense of time scales, $\left\{t_{l}\right\}$ is a sequence of 
real numbers such that $0<t_{1}<\ldots<t_{l} \rightarrow \infty$ as $l \rightarrow \infty$; there exists a positive integer $q$ such that $t_{l+q}=t_{l}+\omega / 2, I_{i k+q}=-I_{i k}, l \in \mathbb{N}, i=1, \ldots, n$. Without loss of generality, we also assume that $[0, \omega / 2)_{\mathbb{T}} \cap\left\{t_{l}: l \in \mathbb{N}\right\}=\left\{t_{1}, \ldots, t_{q}\right\}$.

The initial conditions associated with system (1.1) are of the form

$$
x_{i}(t)=\varphi_{i}(t), \quad t \in[-\tau, 0]_{\mathbb{T}}, \quad \tau=\max _{1 \leq i, j \leq n}\left\{\max _{t \in[0, \omega]_{\mathbb{T}}} \tau_{i j}(t)\right\},
$$

where $\varphi_{i}(t)(i=1, \ldots, n)$ are continuous functions on $[-\tau, 0]_{\mathbb{T}}$.

The main purpose of this paper is to study the existence and global exponential stability of the anti-periodic solutions of system (1.1) by using the method of coincidence degree theory and Lyapunov functional method.

Throughout this paper, we assume that

$\left(\mathrm{H}_{1}\right) \tau_{i j} \geq 0, c_{i j}, d_{i j}, e_{i} \in C(\mathbb{T}, \mathbb{R}), c_{i j}(t+\omega / 2)=c_{i j}(t), d_{i j}(t+\omega / 2)=d_{i j}(t)$, $e_{i}(t+\omega / 2)=-e_{i}(t), i, j=1, \ldots, n$.

$\left(\mathrm{H}_{2}\right) a_{i} \in C(\mathbb{R},(0,+\infty)), a_{i}(-u)=a_{i}(u)$ and there exist positive constants $a_{i}^{m}, a_{i}^{M}$ such that $0<a_{i}^{m} \leq a_{i}(u) \leq a_{i}^{M}$ for all $u \in \mathbb{R}, i=1, \ldots, n$.

$\left(\mathrm{H}_{3}\right) b_{i} \in C(\mathbb{R}, \mathbb{R})$ are delta differentiable, $b_{i}(0)=0, b_{i}(-u)=-b_{i}(u)$ and there exist positive constants $\rho_{i}, \delta_{i}$ such that $0<\rho_{i} \leq b_{i}^{\Delta}(u) \leq \delta_{i}$ for all $u \in \mathbb{R}, i=1, \ldots, n$.

$\left(\mathrm{H}_{4}\right) f_{j}, g_{j} \in C(\mathbb{R}, \mathbb{R}), f_{j}(-u)=-f_{j}(u), g_{j}(-u)=-g_{j}(u)$ and there exist positive constants $L_{j}$ and $P_{j}$ such that

$$
\left|f_{j}(u)-f_{j}(v)\right| \leq L_{j}|u-v| \quad \text { and } \quad\left|g_{j}(u)-g_{j}(v)\right| \leq P_{j}|u-v|
$$

for all $u, v \in \mathbb{R}, j=1, \ldots, n$.

$\left(\mathrm{H}_{5}\right) I_{i k} \in C(\mathbb{R}, \mathbb{R})$ and there exist positive constants $G_{i k}$ such that

$$
\left|I_{i k}(u)-I_{i k}(v)\right| \leq G_{i k}|u-v|
$$

for all $u, v \in \mathbb{R}, k \in \mathbb{N}, i=1, \ldots, n$.

For the sake of convenience, we introduce the following notations:

$$
h^{L}=\max _{t \in[0, \omega]_{\mathbb{T}}}|h(t)|, \quad\|h\|_{2}=\left(\int_{0}^{\omega}|h(t)|^{2} \Delta t\right)^{1 / 2},
$$

where $h$ is an $\omega$-periodic function.

The organization of this paper is as follows. In Section 2, we make some preparations. In Section 3, by using the method of coincidence degree, we obtain the existence of the anti-periodic solutions of system (1.1). In Section 4, we give the criteria of global exponential stability of the anti-periodic solutions of system (1.1). In Section 5, an example is also provided to illustrate the effectiveness of the main results in Sections 3 and 4. The conclusions are drawn in Section 6. 


\section{Preliminaries}

In this section, we shall first recall some basic definitions, lemmas which are used in what follows.

Definition $2.1([5])$. A time scale $\mathbb{T}$ is an arbitrary nonempty closed subset of the real set $\mathbb{R}$ with the topology and ordering inherited from $\mathbb{R}$. The forward and backward jump operators $\sigma, \rho: \mathbb{T} \rightarrow \mathbb{T}$ and the graininess $\mu: \mathbb{T} \rightarrow \mathbb{R}^{+}$are defined, respectively, by

$$
\sigma(t):=\inf \{s \in \mathbb{T}: s>t\}, \quad \rho(t):=\sup \{s \in \mathbb{T}: s<t\}, \quad \mu(t):=\sigma(t)-t .
$$

The point $t \in \mathbb{T}$ is called left-dense, left-scattered, right-dense or right-scattered if $\rho(t)=t, \rho(t)<t, \sigma(t)=t$ or $\sigma(t)>t$, respectively. Points that are rightdense and left-dense at the same time are called dense. If $\mathbb{T}$ has a left-scattered maximum $m$, defined $\mathbb{T}^{k}=\mathbb{T}-\{m\}$; otherwise, set $\mathbb{T}^{k}=\mathbb{T}$.

Definition $2.2([5])$. A vector function $f: \mathbb{T} \rightarrow \mathbb{R}^{n}$ is rd-continuous provided it is continuous at each right-dense point in $\mathbb{T}$ and has a left-sided limit at each left-dense point in $\mathbb{T}$. The set of rd-continuous functions $f: \mathbb{T} \rightarrow \mathbb{R}^{n}$ will be denoted by $C_{\mathrm{rd}}(\mathbb{T})=C_{\mathrm{rd}}\left(\mathbb{T}, \mathbb{R}^{n}\right)$.

Definition $2.3([5])$. For a function $f: \mathbb{T} \rightarrow \mathbb{R}$ (the range $\mathbb{R}$ of $f$ may be actually replaced by Banach space) the (delta) derivative is defined by

$$
f^{\Delta}=\frac{f(\sigma(t))-f(t)}{\sigma(t)-t}
$$

if $f$ is continuous at $t$ and $t$ is right-scattered. If $t$ is not right-scattered then the derivative is defined by

$$
f^{\Delta}=\lim _{s \rightarrow t} \frac{f(\sigma(t))-f(s)}{\sigma(t)-s}=\lim _{s \rightarrow t} \frac{f(t)-f(s)}{t-s}
$$

provided this limit exists.

Definition $2.4([5])$. If $F^{\Delta}(t)=f(t)$, then we define the delta integral by

$$
\int_{a}^{t} f(s) \Delta s=F(t)-F(a) .
$$

Definition $2.5([5])$. A function $p: \mathbb{T} \rightarrow \mathbb{R}$ is said to be regressive provided $1+\mu(t) p(t) \neq 0$ for all $t \in \mathbb{T}^{k}$, where $\mu(t)=\sigma(t)-t$ is the graininess function. The set of all regressive rd-continuous functions $f: \mathbb{T} \rightarrow \mathbb{R}$ is denoted by $\mathcal{R}$ while the set $\mathcal{R}^{+}$is given by $\{f \in \mathcal{R}: 1+\mu(t) f(t)>0\}$ for all $t \in \mathbb{T}$. Let $p \in \mathcal{R}$. The exponential function is defined by

$$
e_{p}(t, s)=\exp \left(\int_{s}^{t} \xi_{\mu(\tau)}(p(\tau)) \Delta \tau\right),
$$

where $\xi_{h(z)}$ is the so-called cylinder transformation. 
Definition 2.6 ([16]). For each $t \in \mathbb{T}$, let $N$ be a neighbourhood of $t$. Then we defined the generalized derivative (or Dini derivative), $D^{+} u^{\Delta}(t)$ to mean that, given $\varepsilon>0$, there exists a right neighbourhood $N(\varepsilon) \subset N$ of $t$ such that

$$
\frac{u(\sigma(t))-u(s)}{\sigma(t)-s}<D^{+} u^{\Delta}(t)+\varepsilon
$$

for each $s \in N(\varepsilon), s>t$.

In case $t$ is right-scattered and $u(t)$ is continuous at $t$, this reduce to

$$
D^{+} u^{\Delta}(t)=\frac{u(\sigma(t))-u(t)}{\sigma(t)-t} .
$$

Similar to [15], we shall first give the definition of anti-periodic function on a time scale as following:

Definition 2.7. We say that a time scale $\mathbb{T}$ is periodic if there exists $p>0$ such that if $t \in \mathbb{T}$, then $t \pm p \in \mathbb{T}$. For $\mathbb{T} \neq \mathbb{R}$, the smallest positive $p$ is called the period of the time scale. Let $\mathbb{T} \neq \mathbb{R}$ be a periodic time scale with period $p$. We say that the function $f: \mathbb{T} \rightarrow \mathbb{R}$ is $\omega / 2$-anti-periodic if there exists a natural number $n$ such that $\omega / 2=n p, f(t+\omega / 2)=-f(t)$ for all $t \in \mathbb{T}$ and $\omega / 2$ is the smallest number such that $f(t+\omega / 2)=-f(t)$. If $\mathbb{T}=\mathbb{R}$, we say that $f$ is $\omega / 2$ anti-periodic if $\omega / 2$ is the smallest positive number such that $f(t+\omega / 2)=-f(t)$ for all $t \in \mathbb{T}$.

Lemma 2.8 ([5]). Let $p, q \in \mathcal{R}$. Then:

(a) $e_{0}(t, s) \equiv 1$ and $e_{p}(t, t) \equiv 1$

(b) $e_{p}(\sigma(t), s)=(1+\mu(t) p(t)) e_{p}(t, s)$;

(c) $1 / e_{p}(t, s)=e_{\ominus p}(t, s)$, where $\ominus p(t)=-p(t) /(1+\mu(t) p(t))$;

(d) $e_{p}(t, s) e_{p}(s, r)=e_{p}(t, r)$;

(e) $e_{p}^{\Delta}(\cdot, s)=p e_{p}(\cdot, s)$.

Lemma $2.9([5])$. Assume that $f, g: \mathbb{T} \rightarrow \mathbb{R}$ are delta differentiable at $t \in \mathbb{T}^{k}$. Then

$$
\begin{aligned}
(f g)^{\Delta}(t) & =f^{\Delta}(t) g(t)+f(\sigma(t)) g^{\Delta}(t)=f(t) g^{\Delta}(t)+f^{\Delta}(t) g(\sigma(t)), \\
f(\sigma(t)) & =f(t)+\mu(t) f^{\Delta}(t) .
\end{aligned}
$$

The following lemmas can be found in [2], [4], [24], respectively.

Lemma 2.10. Let $t_{1}, t_{2} \in[0, \omega]_{\mathbb{T}}$. If $x: \mathbb{T} \rightarrow \mathbb{R}$ is $\omega$-periodic, then

$$
x(t) \leq x\left(t_{1}\right)+\int_{0}^{\omega}\left|x^{\Delta}(s)\right| \Delta s \quad \text { and } \quad x(t) \geq x\left(t_{2}\right)-\int_{0}^{\omega}\left|x^{\Delta}(s)\right| \Delta s .
$$

Lemma 2.11 (Cauchy Schwarz inequality on time scale). Let $a, b \in \mathbb{T}$. For rd-continuous functions $f, g:[a, b]_{\mathbb{T}} \rightarrow \mathbb{R}$ we have

$$
\int_{a}^{b}|f(t)||g(t)| \Delta t \leq\left(\int_{a}^{b}|f(t)|^{2} \Delta t\right)^{1 / 2}\left(\int_{a}^{b}|g(t)|^{2} \Delta t\right)^{1 / 2}
$$


LEMma 2.12 (Mean value theorem, [13]). Let function $f$ be continuous on $[a, b]_{\mathbb{T}}$ and delta differentiable on $[a, b)_{\mathbb{T}}$, then there exist $\xi, \varsigma \in[a, b)_{\mathbb{T}}$ such that

$$
f^{\Delta}(\xi)(b-a) \leq f(b)-f(a) \leq f^{\Delta}(\varsigma)(b-a) .
$$

Definition 2.13. The anti-periodic solution $x^{*}(t)=\left(x_{1}^{*}(t), \ldots, x_{n}^{*}(t)\right)^{T}$ of system (1.1) is said to be globally exponentially stable if there exist positive constants $\lambda$ and $M=M(\lambda) \geq 1$, for any solution $x(t)=\left(x_{1}(t), x_{2}(t), \ldots, x_{n}(t)\right)^{T}$ of system (1.1) with the initial value $\varphi(t)=\left(\varphi_{1}(t), \ldots, \varphi_{n}(t)\right)^{T} \in C\left([-\tau, 0]_{\mathbb{T}}, \mathbb{R}^{n}\right)$, such that

$$
\sum_{i=1}^{n}\left|x_{i}(t)-x_{i}^{*}(t)\right| \leq M(\lambda) e_{\ominus \lambda}(t, \alpha)\left\|\varphi-x^{*}\right\|
$$

where

$$
\left\|\varphi-x^{*}\right\|=\sum_{i=1}^{n} \max _{s \in[-\tau, 0]_{\mathbb{T}}}\left|\varphi_{i}(s)-x_{i}^{*}(s)\right|, \quad \alpha \in[-\tau, 0]_{\mathbb{T}} .
$$

Lemma 2.14. Let $f \in C(\mathbb{T}, \mathbb{R})$ is $\Delta$-differentiable at $t$. Then

$$
\Delta^{r}|f(t)| \leq \operatorname{sign}\left(f^{\sigma}(t)\right) f^{\Delta}(t), \quad \text { where } f^{\sigma}(t)=f(\sigma(t)) .
$$

Proof. Case 1. If $t$ is a right dense point, that is, $\sigma(t)=t$.

$$
\Delta^{r}|f(t)| \leq \operatorname{sign}(f(t)) f^{\Delta}(t)=\operatorname{sign}\left(f^{\sigma}(t)\right) f^{\Delta}(t) .
$$

Case 2. If $t$ is a right scattered point, that is, $\sigma(t)>t$. If $f(t) f^{\sigma}(t)>0$, one can easily have $\operatorname{sign}(f(t))=\operatorname{sign}\left(f^{\sigma}(t)\right)$, so we can obtain

$$
\begin{aligned}
\Delta^{r}|f(t)| & =\frac{\left|f^{\sigma}(t)\right|-|f(t)|}{\mu(t)}=\frac{\operatorname{sign}\left(f^{\sigma}(t)\right) f^{\sigma}(t)-\operatorname{sign}(f(t)) f(t)}{\mu(t)} \\
& =\operatorname{sign}\left(f^{\sigma}(t)\right)\left(\frac{f^{\sigma}(t)-f(t)}{\mu(t)}\right)=\operatorname{sign}\left(f^{\sigma}(t)\right) f^{\Delta}(t) .
\end{aligned}
$$

If $f(t) f^{\sigma}(t) \leq 0$, then one can get $|f(t)| \geq \operatorname{sign}\left(f^{\sigma}(t)\right) f(t)$. Then

$$
\begin{aligned}
\Delta^{r}|f(t)| & =\frac{\left|f^{\sigma}(t)\right|-|f(t)|}{\mu(t)}=\frac{\operatorname{sign}\left(f^{\sigma}(t)\right) f^{\sigma}(t)-|f(t)|}{\mu(t)} \\
& \leq \frac{\operatorname{sign}\left(f^{\sigma}(t)\right) f^{\sigma}(t)-\operatorname{sign}\left(f^{\sigma}(t)\right) f(t)}{\mu(t)} \\
& =\operatorname{sign}\left(f^{\sigma}(t)\right)\left(\frac{f^{\sigma}(t)-f(t)}{\mu(t)}\right)=\operatorname{sign}\left(f^{\sigma}(t)\right) f^{\Delta}(t) .
\end{aligned}
$$

Therefore, by (2.1)-(2.3), one can get $\Delta^{r}|f(t)| \leq \operatorname{sign}\left(f^{\sigma}(t)\right) f^{\Delta}(t)$.

The following fixed point theorem of coincidence degree is crucial in the arguments of our main results.

Lemma $2.15([26])$. Let $\mathbb{X}, \mathbb{Y}$ be two Banach spaces, $\Omega \subset \mathbb{X}$ be open bounded and symmetric with $0 \in \Omega$. Suppose that $L: D(L) \subset \mathbb{X} \rightarrow \mathbb{Y}$ is a linear Fredholm operator of index zero with $D(L) \cap \bar{\Omega} \neq \emptyset$ and $N: \bar{\Omega} \rightarrow \mathbb{Y}$ is L-compact. Further, we also assume that 
(H) $L x-N x \neq \lambda(-L x-N(-x))$ for all $x \in D(L) \cap \partial \Omega, \lambda \in(0,1]$.

Then equation $L x=N x$ has at least one solution on $D(L) \cap \bar{\Omega}$.

\section{Existence of anti-periodic solutions}

TheOREM 3.1. Assume that $\left(\mathrm{H}_{1}\right)-\left(\mathrm{H}_{5}\right)$ hold. Suppose further that

$\left(\mathrm{H}_{6}\right) \Pi=\left(h_{i j}\right)_{n \times n}$ is a nonsingular $M$ matrix, where

$$
h_{i j}=\left\{\begin{array}{cc}
a_{i}^{m}-\delta_{i} a_{i}^{m} a_{i}^{M} \omega-\left(\frac{1}{\omega \rho_{i}}+a_{i}^{m}\right) \sum_{k=1}^{2 q} G_{i k} & \\
-a_{i}^{M}\left(\frac{1}{\rho_{i}}+a_{i}^{m} \omega\right)\left(c_{i j}^{L} L_{j}+d_{i j}^{L} P_{j}\right), & i=j, \\
-a_{i}^{M}\left(\frac{1}{\rho_{i}}+a_{i}^{m} \omega\right)\left(c_{i j}^{L} L_{j}+d_{i j}^{L} P_{j}\right), & i \neq j .
\end{array}\right.
$$

Then system (1.1) has at least one $\omega / 2$-anti-periodic solution.

Proof. Let $C^{k}\left[0, \omega ; t_{1}, \ldots, t_{q}, t_{q+1}, \ldots, t_{2 q}\right]_{\mathbb{T}}=\left\{x:[0, \omega]_{\mathbb{T}} \rightarrow \mathbb{R} \mid x^{k}(t)\right.$ is a piecewise continuous map with first-class discontinuous points in $[0, \omega]_{\mathbb{T}} \cap\left\{t_{l}\right.$ : $l \in \mathbb{N}\}$ and at each discontinuous point it is continuous on the left $\}, k=0,1$. Take

$$
\begin{aligned}
\mathbb{X}=\left\{x \in C\left[0, \omega ; t_{1}, \ldots, t_{q}, t_{q+1}, \ldots, t_{2 q}\right]_{\mathbb{T}}:\right. & \\
& \left.x(t+\omega / 2)=-x(t) \text { for all } t \in[0, \omega / 2]_{\mathbb{T}}\right\}
\end{aligned}
$$

and $\mathbb{Y}=\mathbb{X} \times \mathbb{R}^{n \times q}$, then $X$ and $Y$ are Banach spaces with the norms

$$
\|x\|_{\mathbb{X}}=\sum_{i=1}^{n}\left|x_{i}\right|_{0} \quad \text { and } \quad\|z\|_{\mathbb{Y}}=\|x\|_{\mathbb{X}}+\|y\|
$$

for all $x \in \mathbb{X}, y \in \mathbb{R}^{n \times q}$, respectively, in which $\left|x_{i}\right|_{0}=\max _{t \in[0, \omega]_{\mathbb{T}}}\left|x_{i}(t)\right|, i=1, \ldots, n$, $\|\cdot\|$ is any norm of $\mathbb{R}^{n \times q}$.

Set $L: \operatorname{Dom} L \cap \mathbb{X} \rightarrow \mathbb{Y}, x \rightarrow\left(x^{\Delta}, \Delta x\left(t_{1}\right), \ldots, \Delta x\left(t_{q}\right)\right)$, where

$\operatorname{Dom} L=\left\{x \in C^{1}\left[0, \omega ; t_{1}, \ldots, t_{2 q}\right]_{\mathbb{T}}: x(t+\omega / 2)=-x(t)\right.$ for all $\left.t \in[0, \omega / 2]_{\mathbb{T}}\right\}$, and $N: \mathbb{X} \rightarrow \mathbb{Y}$

$$
N x=\left(\left(\begin{array}{c}
A_{1}(t) \\
\vdots \\
A_{n}(t)
\end{array}\right),\left(\begin{array}{c}
I_{11}\left(x_{1}\left(t_{1}\right)\right) \\
\vdots \\
I_{n 1}\left(x_{n}\left(t_{1}\right)\right)
\end{array}\right),\left(\begin{array}{c}
I_{12}\left(x_{1}\left(t_{2}\right)\right) \\
\vdots \\
I_{n 2}\left(x_{n}\left(t_{2}\right)\right)
\end{array}\right), \ldots,\left(\begin{array}{c}
I_{1 q}\left(x_{1}\left(t_{q}\right)\right) \\
\vdots \\
I_{n q}\left(x_{n}\left(t_{q}\right)\right)
\end{array}\right)\right)
$$


where, for $i=1, \ldots, n$,

$$
\begin{aligned}
A_{i}(t)=-a_{i}\left(x_{i}(t)\right)\left[b_{i}\left(x_{i}(t)\right)-\sum_{j=1}^{n} c_{i j}(t)\right. & f_{j}\left(x_{j}(t)\right) \\
& \left.-\sum_{j=1}^{n} d_{i j}(t) g_{j}\left(x_{j}\left(t-\tau_{i j}(t)\right)\right)-e_{i}(t)\right] .
\end{aligned}
$$

It is easy to see that

$$
\operatorname{Ker} L=\{0\} \quad \text { and } \quad \operatorname{Im} L=\left\{z=\left(f, C_{1}, \ldots, C_{q}\right) \in \mathbb{Y}: \int_{0}^{\omega} f(s) \Delta s=0\right\} \equiv \mathbb{Y} .
$$

Thus $\operatorname{dim} \operatorname{Ker} L=0=\operatorname{codim} \operatorname{Im} L$, and $L$ is a linear Fredholm operator of index zero.

Define the continuous projector $P: \mathbb{X} \rightarrow \operatorname{Ker} L$ and the averaging projector $Q: \mathbb{Y} \rightarrow \mathbb{Y}$ by

$$
P x=\int_{0}^{\omega} x(s) \Delta s=0
$$

and

$$
Q z=Q\left(f, C_{1}, \ldots, C_{q}\right)=\left(\frac{1}{\omega} \int_{0}^{\omega} f(s) \Delta s, 0, \ldots, 0\right) .
$$

Hence $\operatorname{Im} P=\operatorname{Ker} L$ and $\operatorname{Ker} Q=\operatorname{Im} L=\operatorname{Im}(I-Q)$.

Denoting by $L_{P}^{-1}: \operatorname{Im} L \rightarrow \operatorname{Dom}(L) \cap \operatorname{Ker} P$ the inverse of $\left.L\right|_{D(L) \cap \operatorname{Ker} P}$, we have

$$
L_{P}^{-1} z=\int_{0}^{t} f(s) \Delta s+\sum_{t_{k}<t} C_{k}-\frac{1}{2} \int_{0}^{\omega / 2} f(s) \Delta s-\frac{1}{2} \sum_{k=1}^{q} C_{k},
$$

in which $C_{q+i}=-C_{i}$ for all $1 \leq i \leq q$.

Similar to [20], it is not difficult to show that $Q N(\bar{\Omega}), L_{P}^{-1}(I-Q) N(\bar{\Omega})$ are relatively compact for any open bounded set $\Omega \subset \mathbb{X}$. Therefore, $N$ is $L$-compact on $\bar{\Omega}$ for any open bounded set $\Omega \subset \mathbb{X}$.

In order to apply Lemma 2.7, we need to find an appropriate open bounded subset $\Omega$ in $\mathbb{X}$. Corresponding to the operator equation

$$
L x-N x=\lambda(-L x-N(-x)), \quad \lambda \in(0,1],
$$

we have, for $i=1, \ldots, n$,

$$
\begin{cases}x_{i}^{\Delta}(t)=\frac{1}{1+\lambda} G_{i}(t, x)-\frac{\lambda}{1+\lambda} G_{i}(t,-x), & t \in \mathbb{T}^{+}, \\ \Delta x_{i}\left(t_{k}\right)=\frac{1}{1+\lambda} I_{i k}\left(x_{i}\left(t_{k}\right)\right)-\frac{\lambda}{1+\lambda} I_{i k}\left(-x_{i}\left(t_{k}\right)\right), & i=t_{k}, k \in \mathbb{N}, \ldots, n,\end{cases}
$$


where

$$
\begin{aligned}
G_{i}(t, x)=-a_{i}\left(x_{i}(t)\right)\left[b_{i}\left(x_{i}(t)\right)-\sum_{j=1}^{n} c_{i j}(t) f_{j}\left(x_{j}(t)\right)\right. \\
\left.\quad-\sum_{j=1}^{n} d_{i j}(t) g_{j}\left(x_{j}\left(t-\tau_{i j}(t)\right)\right)-e_{i}(t)\right]
\end{aligned}
$$

and

$$
\begin{aligned}
G_{i}(t,-x)=-a_{i}\left(-x_{i}(t)\right)\left[b_{i}\left(-x_{i}(t)\right)\right. & -\sum_{j=1}^{n} c_{i j}(t) f_{j}\left(-x_{j}(t)\right) \\
& \left.-\sum_{j=1}^{n} d_{i j}(t) g_{j}\left(-x_{j}\left(t-\tau_{i j}(t)\right)\right)-e_{i}(t)\right] .
\end{aligned}
$$

Set $t_{0}=t_{0}^{+}=0, t_{2 q+1}=\omega$, in view of $(3.1)$, we get from $\left(\mathrm{H}_{2}\right)-\left(\mathrm{H}_{5}\right)$ and Lemma 2.5 that

$$
\begin{aligned}
& \int_{0}^{\omega}\left|x_{i}^{\Delta}(t)\right| \Delta t=\sum_{k=1}^{2 q+1} \int_{t_{k-1}^{+}}^{t_{k}}\left|x_{i}^{\Delta}(t)\right| \Delta t+\sum_{k=1}^{2 q}\left|I_{i k}\left(x_{i}\left(t_{k}\right)\right)\right| \\
& \leq \int_{0}^{\omega}\left|\frac{1}{1+\lambda} G_{i}(t, x)-\frac{\lambda}{1+\lambda} G_{i}(t,-x)\right| \Delta t+\sum_{k=1}^{2 q}\left|I_{i k}\left(x_{i}\left(t_{k}\right)\right)\right| \\
& \leq {\left[\frac{1}{1+\lambda}+\frac{\lambda}{1+\lambda}\right] \int_{0}^{\omega} \max \left\{\left|G_{i}(t, x)\right|,\left|G_{i}(t,-x)\right|\right\} \Delta t } \\
&+\sum_{k=1}^{2 q}\left|I_{i k}\left(x_{i}\left(t_{k}\right)\right)\right| \\
& \leq a_{i}^{M}\left[\int_{0}^{\omega}\left|b_{i}\left(x_{i}(t)\right)\right| \Delta t+\sum_{j=1}^{n} c_{i j}^{L} \int_{0}^{\omega}\left|f_{j}\left(x_{j}(t)\right)-f_{j}(0)\right| \Delta t\right. \\
&+\sum_{j=1}^{n} d_{i j}^{L} \int_{0}^{\omega}\left|g_{j}\left(x_{j}\left(t-\tau_{i j}\right)\right)-g_{j}(0)\right| \Delta t+e_{i}^{L} \omega \\
&\left.+\sum_{j=1}^{n} c_{i j}^{L} \omega\left|f_{j}(0)\right|+\sum_{j=1}^{n} d_{i j}^{L} \omega\left|g_{j}(0)\right|\right] \\
&+\sum_{k=1}^{2 q}\left|I_{i k}\left(x_{i}\left(t_{k}\right)\right)-I_{i k}(0)\right|+\sum_{k=1}^{2 q}\left|I_{i k}(0)\right| \\
& \leq a_{i}^{M}\left[\delta_{i} \sqrt{\omega}\left\|x_{i}\right\|_{2}+\sum_{j=1}^{n} c_{i j}^{L} L_{j} \sqrt{\omega}\left\|x_{j}\right\|_{2}+\sum_{j=1}^{n} d_{i j}^{L} P_{j} \sqrt{\omega}\left\|x_{j}\right\|_{2}+e_{i}^{L} \omega\right. \\
&\left.+\sum_{j=1}^{n} c_{i j}^{L} \omega\left|f_{j}(0)\right|+\sum_{j=1}^{n} d_{i j}^{L} \omega\left|g_{j}(0)\right|\right]+\sum_{k=1}^{2 q} G_{i k}\left|x_{i}\right|_{0}+\sum_{k=1}^{2 q}\left|I_{i k}(0)\right|
\end{aligned}
$$




$$
\begin{aligned}
= & a_{i}^{M} \delta_{i} \sqrt{\omega}\left\|x_{i}\right\|_{2}+\sum_{k=1}^{2 q} G_{i k}\left|x_{i}\right|_{0}+a_{i}^{M} \sqrt{\omega} \sum_{j=1}^{n}\left(c_{i j}^{L} L_{j}+d_{i j}^{L} P_{j}\right)\left\|x_{j}\right\|_{2} \\
& +a_{i}^{M} e_{i}^{L} \omega+a_{i}^{M} \sum_{j=1}^{n} c_{i j}^{L} \omega\left|f_{j}(0)\right|+a_{i}^{M} \sum_{j=1}^{n} d_{i j}^{L} \omega\left|g_{j}(0)\right|+\sum_{k=1}^{2 q}\left|I_{i k}(0)\right|,
\end{aligned}
$$

for $i=1, \ldots, n$. Integrating (3.1) from 0 to $\omega$, we have from $\left(\mathrm{H}_{2}\right)-\left(\mathrm{H}_{5}\right)$ that

$$
\begin{aligned}
\mid \int_{0}^{\omega} & {\left[\frac{a_{i}\left(x_{i}(t)\right) b_{i}\left(x_{i}(t)\right)}{1+\lambda}-\frac{\lambda a_{i}\left(-x_{i}(t)\right) b_{i}\left(-x_{i}(t)\right)}{1+\lambda}\right] \Delta t \mid } \\
= & \left|\int_{0}^{\omega}\left[\frac{a_{i}\left(x_{i}(t)\right) b_{i}\left(x_{i}(t)\right)}{1+\lambda}+\frac{\lambda a_{i}\left(x_{i}(t)\right) b_{i}\left(x_{i}(t)\right)}{1+\lambda}\right] \Delta t\right| \\
= & \left|\int_{0}^{\omega} a_{i}\left(x_{i}(t)\right) b_{i}\left(x_{i}(t)\right) \Delta t\right| \\
= & \mid \frac{1}{1+\lambda} \int_{0}^{\omega} a_{i}\left(x_{i}(t)\right)\left[\sum_{j=1}^{n} c_{i j}(t) f_{j}\left(x_{j}(t)\right)\right. \\
& \left.+\sum_{j=1}^{n} d_{i j}(t) g_{j}\left(x_{j}\left(t-\tau_{i j}(t)\right)\right)+e_{i}(t)\right] \Delta t \\
& +\frac{\lambda}{1+\lambda} \int_{0}^{\omega} a_{i}\left(-x_{i}(t)\right)\left[-\sum_{j=1}^{n} c_{i j}(t) f_{j}\left(-x_{j}(t)\right)\right. \\
& \left.-\sum_{j=1}^{n} d_{i j}(t) g_{j}\left(-x_{j}\left(t-\tau_{i j}(t)\right)\right)-e_{i}(t)\right] \Delta t \\
& +\frac{1}{1+\lambda} \sum_{k=1}^{2 q} I_{i k}\left(x_{i}\left(t_{k}\right)\right)-\frac{\lambda}{1+\lambda} \sum_{k=1}^{2 q} I_{i k}\left(-x_{i}\left(t_{k}\right)\right) \mid \\
\leq & {\left[\frac{1}{1+\lambda}+\frac{\lambda}{1+\lambda}\right] a_{i}^{M} \int_{0}^{\omega} \max \left\{\mid \sum_{j=1}^{n} c_{i j}(t) f_{j}\left(x_{j}(t)\right)\right.} \\
& +a_{i}^{M} \int_{0}^{\omega}\left[\sum_{j=1}^{n} c_{i j}^{L}\left|f_{j}\left(x_{j}(t)\right)-f_{j}(0)\right|\right. \\
& +\sum_{j=1}^{n} d_{i j}(t) g_{j}\left(x_{j}\left(t-\tau_{i j}(t)\right)\right)+e_{i}(t) \mid, \\
& \left.+\sum_{j=1}^{n} c_{i j}(t) f_{j}\left(x_{j}(t)\right)+\sum_{j=1}^{n} d_{i j}(t) g_{j}\left(x_{j}\left(t-\tau_{i j}(t)\right)\right)-e_{i}(t) \mid\right\} \Delta t \\
& {\left[\frac{1}{1+\lambda}+\frac{\lambda}{1+\lambda}\right] \max \left\{\sum_{k=1}^{2 q}\left|I_{i k}\left(x_{i}\left(t_{k}\right)\right)\right|, \sum_{k=1}^{2 q}\left|I_{i k}\left(-x_{i}\left(t_{k}\right)\right)\right|\right\} } \\
& \\
&
\end{aligned}
$$




$$
\begin{aligned}
& +a_{i}^{M} \sum_{j=1}^{n} c_{i j}^{L} \omega\left|f_{j}(0)\right|+a_{i}^{M} \sum_{j=1}^{n} d_{i j}^{L} \omega\left|g_{j}(0)\right| \\
& +\max \left\{\sum_{k=1}^{2 q}\left|I_{i k}\left(x_{i}\left(t_{k}\right)\right)-I_{i k}(0)\right|, \sum_{k=1}^{2 q}\left|I_{i k}\left(-x_{i}\left(t_{k}\right)\right)-I_{i k}(0)\right|\right\}+\sum_{k=1}^{2 q}\left|I_{i k}(0)\right| \\
\leq & a_{i}^{M}\left[\sum_{j=1}^{n} c_{i j}^{L} L_{j} \sqrt{\omega}\left\|x_{j}\right\|_{2}+\sum_{j=1}^{n} d_{i j}^{L} P_{j} \sqrt{\omega}\left\|x_{j}\right\|_{2}+e_{i}^{L} \omega\right]+a_{i}^{M} \sum_{j=1}^{n} c_{i j}^{L} \omega\left|f_{j}(0)\right| \\
& +a_{i}^{M} \sum_{j=1}^{n} d_{i j}^{L} \omega\left|g_{j}(0)\right|+\sum_{k=1}^{2 q} G_{i k}\left|x_{i}\right|_{0}+\sum_{k=1}^{2 q}\left|I_{i k}(0)\right| \\
= & a_{i}^{M} \sqrt{\omega} \sum_{j=1}^{n}\left(c_{i j}^{L} L_{j}+d_{i j}^{L} P_{j}\right)\left\|x_{j}\right\|_{2}+\sum_{k=1}^{2 q} G_{i k}\left|x_{i}\right|_{0}+a_{i}^{M} e_{i}^{L} \omega \\
& +a_{i}^{M} \sum_{j=1}^{n} c_{i j}^{L} \omega\left|f_{j}(0)\right|+a_{i}^{M} \sum_{j=1}^{n} d_{i j}^{L} \omega\left|g_{j}(0)\right|+\sum_{k=1}^{2 q}\left|I_{i k}(0)\right|
\end{aligned}
$$

for $i=1, \ldots, n$, by Lemma 2.5 and $\left(\mathrm{H}_{3}\right)$, for $i=1, \ldots, n$, we obtain that

$$
\begin{aligned}
& \left|\int_{0}^{\omega} a_{i}\left(x_{i}(t)\right) x_{i}(t) \Delta t\right| \\
& =\frac{a_{i}^{M}}{\rho_{i}} \sqrt{\omega} \sum_{j=1}^{n}\left(c_{i j}^{L} L_{j}+d_{i j}^{L} P_{j}\right)\left\|x_{j}\right\|_{2}+\frac{1}{\rho_{i}} \sum_{k=1}^{2 q} G_{i k}\left|x_{i}\right|_{0}+\frac{a_{i}^{M}}{\rho_{i}} e_{i}^{L} \omega \\
& \quad+\frac{a_{i}^{M}}{\rho_{i}} \sum_{j=1}^{n} c_{i j}^{L} \omega\left|f_{j}(0)\right|+\frac{a_{i}^{M}}{\rho_{i}} \sum_{j=1}^{n} d_{i j}^{L} \omega\left|g_{j}(0)\right|+\frac{1}{\rho_{i}} \sum_{k=1}^{2 q}\left|I_{i k}(0)\right|,
\end{aligned}
$$

From Lemma 2.3 , for any $\zeta_{i}, \eta_{i} \in[0, \omega]_{\mathbb{T}}, i=1, \ldots, n$, we have

$$
\begin{aligned}
& \int_{0}^{\omega} a_{i}\left(x_{i}(t)\right) x_{i}(t) \Delta t \\
& \quad \leq \int_{0}^{\omega} a_{i}\left(x_{i}(t)\right) x_{i}\left(\zeta_{i}\right) \Delta t+\int_{0}^{\omega} a_{i}\left(x_{i}(t)\right)\left(\int_{0}^{\omega}\left|x_{i}^{\Delta}(t)\right| \Delta t\right) \Delta t, \\
& \begin{array}{l}
\int_{0}^{\omega} a_{i}\left(x_{i}(t)\right) x_{i}(t) \Delta t \\
\geq \int_{0}^{\omega} a_{i}\left(x_{i}(t)\right) x_{i}\left(\eta_{i}\right) \Delta t-\int_{0}^{\omega} a_{i}\left(x_{i}(t)\right)\left(\int_{0}^{\omega}\left|x_{i}^{\Delta}(t)\right| \Delta t\right) \Delta t,
\end{array}
\end{aligned}
$$

where $i=1, \ldots, n$. Dividing by $\int_{0}^{\omega} a_{i}\left(x_{i}(t)\right) \Delta t$ on the two sides of (3.4) and (3.5), respectively, we obtain that, for $i=1, \ldots, n$,

$$
x_{i}\left(\zeta_{i}\right) \geq \frac{1}{\int_{0}^{\omega} a_{i}\left(x_{i}(t)\right) \Delta t} \int_{0}^{\omega} a_{i}\left(x_{i}(t)\right) x_{i}(t) \Delta t-\int_{0}^{\omega}\left|x_{i}^{\Delta}(t)\right| \Delta t,
$$




$$
x_{i}\left(\eta_{i}\right) \leq \frac{1}{\int_{0}^{\omega} a_{i}\left(x_{i}(t)\right) \Delta t} \int_{0}^{\omega} a_{i}\left(x_{i}(t)\right) x_{i}(t) \Delta t+\int_{0}^{\omega}\left|x_{i}^{\Delta}(t)\right| \Delta t .
$$

Let $\bar{t}_{i}, \underline{t}_{i} \in[0, \omega]_{\mathbb{T}}$ such that $x_{i}\left(\bar{t}_{i}\right)=\max _{t \in[0, \omega]_{\mathbb{T}}} x_{i}(t), x_{i}\left(\underline{t}_{i}\right)=\min _{t \in[0, \omega]_{\mathbb{T}}} x_{i}(t)$, by the arbitrariness of $\zeta_{i}, \eta_{i}$, we obtain from (3.2), (3.3), (3.6) and (3.7) that

$$
\begin{aligned}
x_{i}\left(\underline{t}_{i}\right) \geq & \frac{1}{\int_{0}^{\omega} a_{i}\left(x_{i}(t)\right) \Delta t} \int_{0}^{\omega} a_{i}\left(x_{i}(t)\right) x_{i}(t) \Delta t-\int_{0}^{\omega}\left|x_{i}^{\Delta}(t)\right| \Delta t \\
\geq & -\frac{1}{\int_{0}^{\omega} a_{i}\left(x_{i}(t)\right) \Delta t}\left|\int_{0}^{\omega} a_{i}\left(x_{i}(t)\right) x_{i}(t) \Delta t\right|-\int_{0}^{\omega}\left|x_{i}^{\Delta}(t)\right| \Delta t \\
\geq & -\frac{1}{a_{i}^{m} \omega}\left[\frac{a_{i}^{M}}{\rho_{i}} \sqrt{\omega} \sum_{j=1}^{n}\left(c_{i j}^{L} L_{j}+d_{i j}^{L} P_{j}\right)\left\|x_{j}\right\|_{2}\right. \\
& +\frac{1}{\rho_{i}} \sum_{k=1}^{2 q} G_{i k}\left|x_{i}\right|_{0}+\frac{a_{i}^{M}}{\rho_{i}} e_{i}^{L} \omega \\
& \left.+\frac{a_{i}^{M}}{\rho_{i}} \sum_{j=1}^{n} c_{i j}^{L} \omega\left|f_{j}(0)\right|+\frac{a_{i}^{M}}{\rho_{i}} \sum_{j=1}^{n} d_{i j}^{L} \omega\left|g_{j}(0)\right|+\frac{1}{\rho_{i}} \sum_{k=1}^{2 q}\left|I_{i k}(0)\right|\right] \\
& -\left[a_{i}^{M} \delta_{i} \sqrt{\omega}\left\|x_{i}\right\|_{2}+\sum_{k=1}^{2 q} G_{i k}\left|x_{i}\right|_{0}\right. \\
& +a_{i}^{M} \sqrt{\omega} \sum_{j=1}^{n}\left(c_{i j}^{L} L_{j}+d_{i j}^{L} P_{j}\right)\left\|x_{j}\right\|_{2}+a_{i}^{M} e_{i}^{L} \omega \\
& \left.+a_{i}^{M} \sum_{j=1}^{n} c_{i j}^{L} \omega\left|f_{j}(0)\right|+a_{i}^{M} \sum_{j=1}^{n} d_{i j}^{L} \omega\left|g_{j}(0)\right|+\sum_{k=1}^{2 q}\left|I_{i k}(0)\right|\right],
\end{aligned}
$$

for $i=1, \ldots, n$ and

$$
\begin{aligned}
x_{i}\left(\bar{t}_{i}\right) \leq & \frac{1}{\int_{0}^{\omega} a_{i}\left(x_{i}(t)\right) \Delta t} \int_{0}^{\omega} a_{i}\left(x_{i}(t)\right) x_{i}(t) \Delta t+\int_{0}^{\omega}\left|x_{i}^{\Delta}(t)\right| \Delta t \\
\leq & \frac{1}{\int_{0}^{\omega} a_{i}\left(x_{i}(t)\right) \Delta t}\left|\int_{0}^{\omega} a_{i}\left(x_{i}(t)\right) x_{i}(t) \Delta t\right|+\int_{0}^{\omega}\left|x_{i}^{\Delta}(t)\right| \Delta t \\
\leq & \frac{1}{a_{i}^{m} \omega}\left[\frac{a_{i}^{M}}{\rho_{i}} \sqrt{\omega} \sum_{j=1}^{n}\left(c_{i j}^{L} L_{j}+d_{i j}^{L} P_{j}\right)\left\|x_{j}\right\|_{2}\right. \\
& +\frac{1}{\rho_{i}} \sum_{k=1}^{2 q} G_{i k}\left|x_{i}\right|_{0}+\frac{a_{i}^{M}}{\rho_{i}} e_{i}^{L} \omega+\frac{a_{i}^{M}}{\rho_{i}} \sum_{j=1}^{n} c_{i j}^{L} \omega\left|f_{j}(0)\right|
\end{aligned}
$$




$$
\begin{aligned}
& \left.+\frac{a_{i}^{M}}{\rho_{i}} \sum_{j=1}^{n} d_{i j}^{L} \omega\left|g_{j}(0)\right|+\frac{1}{\rho_{i}} \sum_{k=1}^{2 q}\left|I_{i k}(0)\right|\right] \\
& +\left[a_{i}^{M} \delta_{i} \sqrt{\omega}\left\|x_{i}\right\|_{2}+\sum_{k=1}^{2 q} G_{i k}\left|x_{i}\right|_{0}\right. \\
& +a_{i}^{M} \sqrt{\omega} \sum_{j=1}^{n}\left(c_{i j}^{L} L_{j}+d_{i j}^{L} P_{j}\right)\left\|x_{j}\right\|_{2}+a_{i}^{M} e_{i}^{L} \omega \\
& \left.+a_{i}^{M} \sum_{j=1}^{n} c_{i j}^{L} \omega\left|f_{j}(0)\right|+a_{i}^{M} \sum_{j=1}^{n} d_{i j}^{L} \omega\left|g_{j}(0)\right|+\sum_{k=1}^{2 q}\left|I_{i k}(0)\right|\right]
\end{aligned}
$$

for $i=1, \ldots, n$. Thus, we have from (3.8) and (3.9) that

$$
\begin{aligned}
\left|x_{i}\right|_{0}= & \max _{t \in[0, \omega]_{\mathbb{T}}}\left|x_{i}(t)\right| \leq \frac{1}{a_{i}^{m} \omega}\left[\frac{a_{i}^{M}}{\rho_{i}} \sqrt{\omega} \sum_{j=1}^{n}\left(c_{i j}^{L} L_{j}+d_{i j}^{L} P_{j}\right)\left\|x_{j}\right\|_{2}\right. \\
& +\frac{1}{\rho_{i}} \sum_{k=1}^{2 q} G_{i k}\left|x_{i}\right|_{0}+\frac{a_{i}^{M}}{\rho_{i}} e_{i}^{L} \omega+\frac{a_{i}^{M}}{\rho_{i}} \sum_{j=1}^{n} c_{i j}^{L} \omega\left|f_{j}(0)\right| \\
& \left.+\frac{a_{i}^{M}}{\rho_{i}} \sum_{j=1}^{n} d_{i j}^{L} \omega\left|g_{j}(0)\right|+\frac{1}{\rho_{i}} \sum_{k=1}^{2 q}\left|I_{i k}(0)\right|\right] \\
& +\left[a_{i}^{M} \delta_{i} \sqrt{\omega}\left\|x_{i}\right\|_{2}+\sum_{k=1}^{2 q} G_{i k}\left|x_{i}\right|_{0}\right. \\
& +a_{i}^{M} \sqrt{\omega} \sum_{j=1}^{n}\left(c_{i j}^{L} L_{j}+d_{i j}^{L} P_{j}\right)\left\|x_{j}\right\|_{2}+a_{i}^{M} e_{i}^{L} \omega \\
& \left.+a_{i}^{M} \sum_{j=1}^{n} c_{i j}^{L} \omega\left|f_{j}(0)\right|+a_{i}^{M} \sum_{j=1}^{n} d_{i j}^{L} \omega\left|g_{j}(0)\right|+\sum_{k=1}^{2 q}\left|I_{i k}(0)\right|\right]
\end{aligned}
$$

for $i=1, \ldots, n$. In addition, we have that

$$
\left\|x_{i}\right\|_{2}=\left(\int_{0}^{\omega}\left|x_{i}(s)\right|^{2} \Delta s\right)^{1 / 2} \leq \sqrt{\omega} \max _{t \in[0, \omega]_{\mathbb{T}}}\left|x_{i}(t)\right|=\sqrt{\omega}\left|x_{i}\right|_{0}, \quad i=1, \ldots, n .
$$

By (3.10), we have

$$
\begin{aligned}
a_{i}^{m} \omega\left|x_{i}\right|_{0} \leq & {\left[\frac{a_{i}^{M}}{\rho_{i}} \sqrt{\omega} \sum_{j=1}^{n}\left(c_{i j}^{L} L_{j}+d_{i j}^{L} P_{j}\right)\left\|x_{j}\right\|_{2}+\frac{1}{\rho_{i}} \sum_{k=1}^{2 q} G_{i k}\left|x_{i}\right|_{0}+\frac{a_{i}^{M}}{\rho_{i}} e_{i}^{L} \omega\right.} \\
& \left.+\frac{a_{i}^{M}}{\rho_{i}} \sum_{j=1}^{n} c_{i j}^{L} \omega\left|f_{j}(0)\right|+\frac{a_{i}^{M}}{\rho_{i}} \sum_{j=1}^{n} d_{i j}^{L} \omega\left|g_{j}(0)\right|+\frac{1}{\rho_{i}} \sum_{k=1}^{2 q}\left|I_{i k}(0)\right|\right] \\
& +a_{i}^{m} \omega\left[a_{i}^{M} \delta_{i} \sqrt{\omega}\left\|x_{i}\right\|_{2}+\sum_{k=1}^{2 q} G_{i k}\left|x_{i}\right|_{0}\right.
\end{aligned}
$$




$$
\begin{aligned}
& +a_{i}^{M} \sqrt{\omega} \sum_{j=1}^{n}\left(c_{i j}^{L} L_{j}+d_{i j}^{L} P_{j}\right)\left\|x_{j}\right\|_{2}+a_{i}^{M} e_{i}^{L} \omega \\
& \left.+a_{i}^{M} \sum_{j=1}^{n} c_{i j}^{L} \omega\left|f_{j}(0)\right|+a_{i}^{M} \sum_{j=1}^{n} d_{i j}^{L} \omega\left|g_{j}(0)\right|+\sum_{k=1}^{2 q}\left|I_{i k}(0)\right|\right] \\
& \leq\left[\frac{a_{i}^{M}}{\rho_{i}} \omega \sum_{j=1}^{n}\left(c_{i j}^{L} L_{j}+d_{i j}^{L} P_{j}\right)\left|x_{j}\right|_{0}+\frac{1}{\rho_{i}} \sum_{k=1}^{2 q} G_{i k}\left|x_{i}\right|_{0}+\frac{a_{i}^{M}}{\rho_{i}} e_{i}^{L} \omega\right. \\
& \left.+\frac{a_{i}^{M}}{\rho_{i}} \sum_{j=1}^{n} c_{i j}^{L} \omega\left|f_{j}(0)\right|+\frac{a_{i}^{M}}{\rho_{i}} \sum_{j=1}^{n} d_{i j}^{L} \omega\left|g_{j}(0)\right|+\frac{1}{\rho_{i}} \sum_{k=1}^{2 q}\left|I_{i k}(0)\right|\right] \\
& +a_{i}^{m} \omega\left[a_{i}^{M} \delta_{i} \omega\left|x_{i}\right|_{0}+\sum_{k=1}^{2 q} G_{i k}\left|x_{i}\right|_{0}+a_{i}^{M} \omega \sum_{j=1}^{n}\left(c_{i j}^{L} L_{j}+d_{i j}^{L} P_{j}\right)\left|x_{j}\right|_{0}\right. \\
& \left.+a_{i}^{M} e_{i}^{L} \omega+a_{i}^{M} \sum_{j=1}^{n} c_{i j}^{L} \omega\left|f_{j}(0)\right|+a_{i}^{M} \sum_{j=1}^{n} d_{i j}^{L} \omega\left|g_{j}(0)\right|+\sum_{k=1}^{2 q}\left|I_{i k}(0)\right|\right]
\end{aligned}
$$

where $i=1,2, \ldots, n$. That is,

$$
\begin{aligned}
&(3.11) \quad\left[a_{i}^{m}-\delta_{i} a_{i}^{m} a_{i}^{M} \omega\right.\left.-\frac{1}{\omega \rho_{i}} \sum_{k=1}^{2 q} G_{i k}-a_{i}^{m} \sum_{k=1}^{2 q} G_{i k}\right]\left|x_{i}\right|_{0} \\
&-a_{i}^{M}\left(\frac{1}{\rho_{i}}+a_{i}^{m} \omega\right) \sum_{j=1}^{n}\left(c_{i j}^{L} L_{j}+d_{i j}^{L} P_{j}\right)\left|x_{j}\right|_{0} \leq \frac{1}{\omega}\left(\frac{1}{\rho_{i}}+a_{i}^{m} \omega\right)\left[a_{i}^{M} \sum_{j=1}^{n} c_{i j}^{L} \omega\left|f_{j}(0)\right|\right. \\
&\left.+a_{i}^{M} \sum_{j=1}^{n} d_{i j}^{L} \omega\left|g_{j}(0)\right|+a_{i}^{M} e_{i}^{L} \omega+\sum_{k=1}^{2 q}\left|I_{i k}(0)\right|\right]=D_{i}
\end{aligned}
$$

for $i=1, \ldots, n$. Denote $|x|_{0}=\left(\left|x_{1}\right|_{0}, \ldots,\left|x_{n}\right|_{0}\right)^{T}$ and $D=\left(D_{1}, \ldots, D_{n}\right)^{T}$. Then (3.11) can be rewritten in the matrix form $\Pi|x|_{0} \leq D$.

From the conditions of Theorem 3.1, $\Pi$ is a nonsingular $M$ matrix, hence

$$
|x|_{0} \leq \Pi^{-1} D \triangleq\left(M_{1}, \ldots, M_{n}\right)^{T} .
$$

Let $M=\sum_{i=1}^{n} M_{i}+1$ (clearly, $M$ is independent of $\lambda$ ). Take $\Omega=\{x \in \mathbb{X}$ : $\left.\|x\|_{\mathbb{X}}<M\right\}$. It is clear that $\Omega$ satisfies all the requirements in Lemma 2.7 and the condition $(\mathrm{H})$ is satisfied. In view of all the discussions above, we conclude from Lemma 2.7 that system (1.1) has at least one $\omega / 2$-anti-periodic solution. This completes the proof.

\section{Global exponential stability of the anti-periodic solution}

Suppose that $x^{*}(t)=\left(x_{1}^{*}(t), \ldots, x_{n}^{*}(t)\right)^{T}$ is an $\omega / 2$-anti-periodic solution of system (1.1). In this section, we will construct some suitable Lyapunov functions to study the global exponential stability of this anti-periodic solution. 
TheOrem 4.1. Assume that $\left(\mathrm{H}_{1}\right)-\left(\mathrm{H}_{6}\right)$ hold and $\tau_{i j}(t) \equiv \tau_{i j}(i, j=1, \ldots, n)$ are nonnegative constants. Suppose further that

$\left(\mathrm{H}_{7}\right)$ There exist positive constants $f_{j}^{M}, g_{j}^{M}$ such that $\left|f_{j}(u)\right| \leq f_{j}^{M}$ and $\left|g_{j}(u)\right| \leq g_{j}^{M}$ for all $u \in \mathbb{R}, j=1, \ldots, n$.

$\left(\mathrm{H}_{8}\right)$ There exist positive constants $\nu_{i}$ such that

$$
\left|a_{i}(u)-a_{i}(v)\right| \leq \nu_{i}|u-v| \quad \text { for all } u, v \in \mathbb{R}, i=1, \ldots, n .
$$

$\left(\mathrm{H}_{9}\right)$ There exist positive constants $l_{i}$ such that

$\left(a_{i}(u) b_{i}(u)-a_{i}(v) b_{i}(v)\right)(u-v) \geq 0 \quad$ and $\quad\left|a_{i}(u) b_{i}(u)-a_{i}(v) b_{i}(v)\right| \geq l_{i}|u-v|$ for all $u, v \in \mathbb{R}, i=1, \ldots, n$.

$\left(\mathrm{H}_{10}\right)$ There exists a positive constant $\varepsilon$ such that $\max _{t \in[0, \omega / 2]_{\mathbb{T}}} \Theta_{i}(\varepsilon, t)<0$, where

$$
\begin{aligned}
\Theta_{i}(\varepsilon, t)= & \varepsilon+(1+\varepsilon \mu(t))\left(2 \mu(t) \zeta_{i}^{2}-\zeta_{i}\right)+\sum_{j=1}^{n} a_{i}^{M}\left[\left(1+2 \mu(t) \zeta_{i}\right) c_{i j}^{L} L_{j}\right. \\
& +\left(1+\varepsilon \mu\left(t+\tau_{i j}\right)\right)\left(1+2 \mu\left(t+\tau_{i j}\right) \zeta_{i}\right) e_{\varepsilon}\left(t+\tau_{i j}, t\right) d_{i j}^{L} P_{j}
\end{aligned}
$$

where

$$
\zeta_{i}=l_{i}-\nu_{i}\left(\sum_{j=1}^{n} c_{i j}^{L} f_{j}^{M}+\sum_{j=1}^{n} d_{i j}^{L} g_{j}^{M}+e_{i}^{L}\right),
$$

for $i=1, \ldots, n$.

$\left(\mathrm{H}_{11}\right)$ The impulsive operators $I_{i k}\left(x_{i}(t)\right)$ satisfy

$$
I_{i k}\left(x_{i}\left(t_{k}\right)\right)=-\gamma_{i k} x_{i}\left(t_{k}\right), \quad 0 \leq \gamma_{i k} \leq 2, i=1, \ldots, n, k \in \mathbb{N} .
$$

Then the $\omega / 2$-anti-periodic solution of system (1.1) is globally exponentially stable.

Proof. According to Theorem 3.1, we know that system (1.1) has an $\omega / 2$ anti-periodic solution $x^{*}(t)=\left(x_{1}^{*}(t), \ldots, x_{n}^{*}(t)\right)^{T}$ with initial value $x^{*}(s), s \in$ $[-\tau, 0]_{\mathbb{T}}$, suppose that $x(t)=\left(x_{1}(t), \ldots, x_{n}(t)\right)^{T}$ is an arbitrary solution of system (1.1) with initial value $\varphi(s), s \in[-\tau, 0]_{\mathbb{T}}$. Then it follows from system (1.1) that

$$
\left\{\begin{array}{cl}
\left(x_{i}(t)-x_{i}^{*}(t)\right)^{\Delta}=-\left[a_{i}\left(x_{i}(t)\right) b_{i}\left(x_{i}(t)\right)-a_{i}\left(x_{i}^{*}(t)\right) b_{i}\left(x_{i}^{*}(t)\right)\right] & \\
+a_{i}\left(x_{i}(t)\right)\left[\sum_{j=1}^{n} c_{i j}(t) f_{j}\left(x_{j}(t)\right)+\sum_{j=1}^{n} d_{i j}(t) g_{j}\left(x_{j}\left(t-\tau_{i j}\right)\right)+e_{i}(t)\right] \\
-a_{i}\left(x_{i}^{*}(t)\right)\left[\sum_{j=1}^{n} c_{i j}(t) f_{j}\left(x_{j}^{*}(t)\right)+\sum_{j=1}^{n} d_{i j}(t) g_{j}\left(x_{j}^{*}\left(t-\tau_{i j}\right)\right)+e_{i}(t)\right], \\
& t \in \mathbb{T}^{+}, t \neq t_{k}, k \in \mathbb{N}, \\
\Delta\left(x_{i}\left(t_{k}\right)-x_{i}^{*}\left(t_{k}\right)\right)=-\gamma_{i k}\left(x_{i}\left(t_{k}\right)-x_{i}^{*}\left(t_{k}\right)\right), & i=1, \ldots, n .
\end{array}\right.
$$


In view of system (4.1), for $t \in \mathbb{T}^{+}, t \neq t_{k}, k \in \mathbb{N}, i=1, \ldots, n$, we have

$$
\begin{aligned}
\left(x_{i}(t)-x_{i}^{*}(t)\right)^{\Delta}= & -\left[a_{i}\left(x_{i}(t)\right) b_{i}\left(x_{i}(t)\right)-a_{i}\left(x_{i}^{*}(t)\right) b_{i}\left(x_{i}^{*}(t)\right)\right] \\
& +\left[a_{i}\left(x_{i}(t)\right)-a_{i}\left(x_{i}^{*}(t)\right)\right] \sum_{j=1}^{n} c_{i j}(t) f_{j}\left(x_{j}(t)\right) \\
& +a_{i}\left(x_{i}^{*}(t)\right) \sum_{j=1}^{n} c_{i j}(t)\left[f_{j}\left(x_{j}(t)\right)-f_{j}\left(x_{j}^{*}(t)\right)\right] \\
& +\left[a_{i}\left(x_{i}(t)\right)-a_{i}\left(x_{i}^{*}(t)\right)\right] \sum_{j=1}^{n} d_{i j}(t) g_{j}\left(x_{j}\left(t-\tau_{i j}\right)\right) \\
& +a_{i}\left(x_{i}^{*}(t)\right) \sum_{j=1}^{n} d_{i j}(t)\left[g_{j}\left(x_{j}\left(t-\tau_{i j}\right)\right)-g_{j}\left(x_{j}^{*}\left(t-\tau_{i j}\right)\right)\right] \\
& +\left[a_{i}\left(x_{i}(t)\right)-a_{i}\left(x_{i}^{*}(t)\right)\right] e_{i}(t) .
\end{aligned}
$$

Denote $y_{i}(t)=x_{i}(t)-x_{i}^{*}(t), i=1, \ldots, n$. Hence we can obtain from $\left(\mathrm{H}_{7}\right)-\left(\mathrm{H}_{9}\right)$ and Lemma 2.6 that

$$
\begin{aligned}
D^{+}\left|y_{i}(t)\right|^{\Delta} \leq & \operatorname{sign}\left(y_{i}^{\sigma}(t)\right) y_{i}^{\Delta}(t) \\
\leq & \left(-l_{i}+\nu_{i}\left(\sum_{j=1}^{n} c_{i j}^{L} f_{j}^{M}+\sum_{j=1}^{n} d_{i j}^{L} g_{j}^{M}+e_{i}^{L}\right)\right)\left|y_{i}^{\sigma}(t)-\mu(t) y_{i}^{\Delta}(t)\right| \\
& +a_{i}^{M} \sum_{j=1}^{n} c_{i j}^{L} L_{j}\left|y_{j}(t)\right|+a_{i}^{M} \sum_{j=1}^{n} d_{i j}^{L} P_{j}\left|y_{j}\left(t-\tau_{i j}\right)\right| \\
\leq & \left(-l_{i}+\nu_{i}\left(\sum_{j=1}^{n} c_{i j}^{L} f_{j}^{M}+\sum_{j=1}^{n} d_{i j}^{L} g_{j}^{M}+e_{i}^{L}\right)\right)\left|y_{i}^{\sigma}(t)\right| \\
& +\mu(t)\left(-l_{i}+\nu_{i}\left(\sum_{j=1}^{n} c_{i j}^{L} f_{j}^{M}+\sum_{j=1}^{n} d_{i j}^{L} g_{j}^{M}+e_{i}^{L}\right)\right)\left|y_{i}^{\Delta}(t)\right| \\
& +a_{i}^{M} \sum_{j=1}^{n} c_{i j}^{L} L_{j}\left|y_{j}(t)\right|+a_{i}^{M} \sum_{j=1}^{n} d_{i j}^{L} P_{j}\left|y_{j}\left(t-\tau_{i j}\right)\right| \\
\leq & -\zeta_{i}\left|y_{i}(t)\right|+2 \mu(t) \zeta_{i}\left|y_{i}^{\Delta}(t)\right| \\
& +a_{i}^{M} \sum_{j=1}^{n} c_{i j}^{L} L_{j}\left|y_{j}(t)\right|+a_{i}^{M} \sum_{j=1}^{n} d_{i j}^{L} P_{j}\left|y_{j}\left(t-\tau_{i j}\right)\right| \\
\leq & \left(2 \mu(t) \zeta_{i}^{2}-\zeta_{i}\right)\left|y_{i}(t)\right| \\
& +\left(1+2 \mu(t) \zeta_{i}\right) a_{i}^{M}\left[\sum_{j=1}^{n} c_{i j}^{L} L_{j}\left|y_{j}(t)\right|+\sum_{j=1}^{n} d_{i j}^{L} P_{j}\left|y_{j}\left(t-\tau_{i j}\right)\right|\right]
\end{aligned}
$$

or $i=1, \ldots, n$. And we have from $\left(\mathrm{H}_{11}\right)$ that

$$
\left|y_{i}\left(t_{k}^{+}\right)\right|=\left|1-\gamma_{i k}\right|\left|y_{i}\left(t_{k}\right)\right| \leq\left|y_{i}\left(t_{k}\right)\right|, \quad i=1, \ldots, n, k \in \mathbb{N} .
$$


For any $\alpha \in[-\tau, 0]_{\mathbb{T}}$, we construct the Lyapunov functional

$$
\begin{aligned}
V(t) & =V_{1}(t)+V_{2}(t), \\
V_{1}(t) & =\sum_{i=1}^{n} e_{\varepsilon}(t, \alpha)\left|y_{i}(t)\right|, \\
V_{2}(t) & =\sum_{i=1}^{n} \sum_{j=1}^{n} \int_{t-\tau_{i j}}^{t}\left(1+\varepsilon \mu\left(s+\tau_{i j}\right)\right) e_{\varepsilon}\left(s+\tau_{i j}, \alpha\right)\left(1+2 \mu\left(s+\tau_{i j}\right)\right. \\
& \times \zeta_{i} a_{i}^{M} d_{i j}^{L} P_{j}\left|y_{j}(s)\right| \Delta s .
\end{aligned}
$$

For $t \in \mathbb{T}^{+}, t \neq t_{k}, k \in \mathbb{N}$, calculating the delta derivative $D^{+} V(t)^{\Delta}$ of $V(t)$ along system (4.1), we can get

$$
\begin{aligned}
D^{+} V_{1}^{\Delta}(t)= & \sum_{i=1}^{n}\left[\varepsilon e_{\varepsilon}(t, \alpha)\left|y_{i}(t)\right|+e_{\varepsilon}(\sigma(t), \alpha) D^{+}\left|y_{i}(t)\right|^{\Delta}\right] \\
\leq & \sum_{i=1}^{n}\left\{\varepsilon e_{\varepsilon}(t, \alpha)\left|y_{i}(t)\right|+(1+\varepsilon \mu(t)) e_{\varepsilon}(t, \alpha)\left(\left(2 \mu(t) \zeta_{i}^{2}-\zeta_{i}\right)\left|y_{i}(t)\right|\right.\right. \\
& \left.\left.\left.+\left(1+2 \mu(t) \zeta_{i}\right) a_{i}^{M}\left[\sum_{j=1}^{n} c_{i j}^{L} L_{j}\left|y_{j}(t)\right|+\sum_{j=1}^{n} d_{i j}^{L} P_{j} \mid y_{j}\left(t-\tau_{i j}\right)\right]\right]\right)\right\} \\
\leq & \sum_{i=1}^{n}\left[\varepsilon+(1+\varepsilon \mu(t))\left(2 \mu(t) \zeta_{i}^{2}-\zeta_{i}\right)\right] e_{\varepsilon}(t, \alpha)\left|y_{i}(t)\right| \\
& +(1+\varepsilon \mu(t)) e_{\varepsilon}(t, \alpha) \\
& \times \sum_{i=1}^{n} \sum_{j=1}^{n}\left(1+2 \mu(t) \zeta_{i}\right) a_{i}^{M}\left[c_{i j}^{L} L_{j}\left|y_{j}(t)\right|+d_{i j}^{L} P_{j}\left|y_{j}\left(t-\tau_{i j}\right)\right|\right]
\end{aligned}
$$

and

$$
\begin{aligned}
D^{+} V_{2}^{\Delta}(t) & \\
\leq \sum_{i=1}^{n} \sum_{j=1}^{n}( & \left.+\varepsilon \mu\left(t+\tau_{i j}\right)\right)\left(1+2 \mu\left(t+\tau_{i j}\right) \zeta_{i}\right) e_{\varepsilon}\left(t+\tau_{i j}, \alpha\right) a_{i}^{M} d_{i j}^{L} P_{j}\left|y_{j}(t)\right| \\
& -(1+\varepsilon \mu(t)) e_{\varepsilon}(t, \alpha) \sum_{i=1}^{n} \sum_{j=1}^{n}\left(1+2 \mu(t) \zeta_{i}\right) a_{i}^{M} d_{i j}^{L} P_{j}\left|y_{j}\left(t-\tau_{i j}\right)\right| .
\end{aligned}
$$

By assumption $\left(\mathrm{H}_{10}\right)$, it concludes that

$$
\begin{aligned}
& D^{+}\left(V^{\Delta}(t)\right)=D^{+} V_{1}^{\Delta}(t)+D^{+} V_{2}^{\Delta}(t) \\
& \leq \sum_{i=1}^{n}\left[\varepsilon+(1+\varepsilon \mu(t))\left(2 \mu(t) \zeta_{i}^{2}-\zeta_{i}\right)\right] e_{\varepsilon}(t, \alpha)\left|y_{i}(t)\right| \\
& \quad+(1+\varepsilon \mu(t)) e_{\varepsilon}(t, \alpha) \sum_{i=1}^{n} \sum_{j=1}^{n}\left(1+2 \mu(t) \zeta_{i}\right) a_{i}^{M} c_{i j}^{L} L_{j}\left|y_{j}(t)\right|
\end{aligned}
$$




$$
\begin{aligned}
& +\sum_{i=1}^{n} \sum_{j=1}^{n}\left(1+\varepsilon \mu\left(t+\tau_{i j}\right)\right)\left(1+2 \mu\left(t+\tau_{i j}\right) \zeta_{i}\right) e_{\varepsilon}\left(t+\tau_{i j}, \alpha\right) a_{i}^{M} d_{i j}^{L} P_{j}\left|y_{j}(t)\right| \\
= & \sum_{i=1}^{n}\left\{\varepsilon+(1+\varepsilon \mu(t))\left(2 \mu(t) \zeta_{i}^{2}-\zeta_{i}\right)+\sum_{j=1}^{n} a_{i}^{M}\left[\left(1+2 \mu(t) \zeta_{i}\right) c_{i j}^{L} L_{j}\right.\right. \\
& \left.\left.+\left(1+\varepsilon \mu\left(t+\tau_{i j}\right)\right)\left(1+2 \mu\left(t+\tau_{i j}\right) \zeta_{i}\right) e_{\varepsilon}\left(t+\tau_{i j}, t\right) d_{i j}^{L} P_{j}\right]\right\} e_{\varepsilon}(t, \alpha)\left|y_{i}(t)\right| \leq 0,
\end{aligned}
$$

for $t \in \mathbb{T}^{+}, t \neq t_{k}, k \in \mathbb{N}$. Also, for $k \in \mathbb{N}$,

$$
\begin{aligned}
V\left(t_{k}^{+}\right)=V_{1}\left(t_{k}^{+}\right)+V_{2}\left(t_{k}^{+}\right) \\
=\sum_{i=1}^{n} e_{\varepsilon}\left(t_{k}^{+}, \alpha\right)\left|y_{i}\left(t_{k}^{+}\right)\right| \\
\quad+\sum_{i=1}^{n} \sum_{j=1}^{n} \int_{t_{k}^{+}-\tau_{i j}}^{t_{k}^{+}}\left(1+\varepsilon \mu\left(s+\tau_{i j}\right)\right) e_{\varepsilon}\left(s+\tau_{i j}, \alpha\right)\left(1+2 \mu\left(s+\tau_{i j}\right) \zeta_{i} a_{i}^{M} d_{i j}^{L} P_{j}\left|y_{j}(s)\right| \Delta s\right. \\
\leq \sum_{i=1}^{n} e_{\varepsilon}\left(t_{k}, \alpha\right)\left|y_{i}\left(t_{k}\right)\right| \\
\quad+\sum_{i=1}^{n} \sum_{j=1}^{n} \int_{t_{k}-\tau_{i j}}^{t_{k}}\left(1+\varepsilon \mu\left(s+\tau_{i j}\right)\right) e_{\varepsilon}\left(s+\tau_{i j}, \alpha\right)\left(1+2 \mu\left(s+\tau_{i j}\right) \zeta_{i} a_{i}^{M} d_{i j}^{L} P_{j}\left|y_{j}(s)\right| \Delta s\right. \\
=V\left(t_{k}\right) .
\end{aligned}
$$

It follows that $V(t) \leq V(0)$ for all $t \in \mathbb{T}^{+}$. On the other hand, we have

$$
\begin{aligned}
V(0)= & V_{1}(0)+V_{2}(0) \\
= & \sum_{i=1}^{n} e_{\varepsilon}(0, \alpha)\left|y_{i}(0)\right| \\
& +\sum_{i=1}^{n} \sum_{j=1}^{n} \int_{-\tau_{i j}}^{0}\left(1+\varepsilon \mu\left(s+\tau_{i j}\right)\right) e_{\varepsilon}\left(s+\tau_{i j}, \alpha\right) \\
& \times\left(1+2 \mu\left(s+\tau_{i j}\right) \zeta_{i} a_{i}^{M} d_{i j}^{L} P_{j}\left|y_{j}(s)\right| \Delta s\right. \\
\leq & \sum_{i=1}^{n}\left\{e_{\varepsilon}(0, \alpha)+\sum_{j=1}^{n} \int_{-\tau_{j i}}^{0}\left(1+\varepsilon \mu\left(s+\tau_{i j}\right)\right) e_{\varepsilon}\left(s+\tau_{i j}, \alpha\right)\right. \\
& \times\left(1+2 \mu\left(s+\tau_{i j}\right) \zeta_{j} a_{i}^{M} d_{j i}^{L} P_{i} \Delta s\right\} \times \max _{s \in[-\tau, 0]_{\mathbb{T}}}\left|y_{i}(s)\right| \\
\leq & \Gamma(\varepsilon) \sum_{i=1}^{n} \max _{s \in[-\tau, 0]_{\mathbb{T}}}\left|\varphi_{i}(s)-x_{i}^{*}(s)\right|,
\end{aligned}
$$


where

$$
\begin{array}{r}
\Gamma(\varepsilon)=\max _{1 \leq i \leq n}\left\{\operatorname { m a x } _ { \alpha \in [ - \tau , 0 ] _ { \mathbb { T } } } \left\{e_{\varepsilon}(0, \alpha)+\sum_{j=1}^{n} \int_{-\tau_{j i}}^{0}\left(1+\varepsilon \mu\left(s+\tau_{i j}\right)\right) e_{\varepsilon}\left(s+\tau_{i j}, \alpha\right)\right.\right. \\
\left.\times\left(1+2 \mu\left(s+\tau_{i j}\right) \zeta_{j} a_{i}^{M} d_{j i}^{L} P_{i} \Delta s\right\}\right\} .
\end{array}
$$

It is obvious that

$$
\sum_{i=1}^{n} e_{\varepsilon}(t, \alpha)\left|x_{i}(t)-x_{i}^{*}(t)\right| \leq V(t) \leq V(0) \leq \Gamma(\varepsilon) \sum_{i=1}^{n} \max _{s \in[-\tau, 0]_{\mathbb{T}}}\left|\varphi_{i}(s)-x_{i}^{*}(s)\right| .
$$

So we can finally get

$$
\begin{aligned}
\sum_{i=1}^{n} \mid x_{i}(t) & -x_{i}^{*}(t) \mid \\
& \leq \Gamma(\varepsilon) e_{\ominus \varepsilon}(t, \alpha) \sum_{i=1}^{n} \max _{s \in[-\tau, 0]_{\mathbb{T}}}\left|\varphi_{i}(s)-x_{i}^{*}(s)\right|=\Gamma(\varepsilon) e_{\ominus \varepsilon}(t, \alpha)\left\|\varphi-x^{*}\right\| .
\end{aligned}
$$

Since $\Gamma(\varepsilon) \geq 1$, from Definition 2.8, the $\omega / 2$-anti-periodic solution of system (1.1) is globally exponential stable. This completes the proof.

\section{An example}

EXAMPLE 5.1. Consider the following impulsive Cohen-Grossberg neural networks

$$
\left\{\begin{aligned}
x_{i}^{\Delta}(t)=-a_{i}\left(x_{i}(t)\right)\left[b_{i}\left(x_{i}(t)\right)-\sum_{j=1}^{n} c_{i j}(t) f_{j}\right. & \\
\left.\left(x_{j}(t)\right)-\sum_{j=1}^{n} d_{i j}(t) g_{j}\left(x_{j}\left(t-\tau_{i j}\right)\right)-e_{i}(t)\right] & \\
& t \in \mathbb{T}^{+}, t \neq t_{k}, k \in \mathbb{N}, \\
\Delta x_{i}\left(t_{k}\right)=x_{i}\left(t_{k}^{+}\right)-x_{i}\left(t_{k}^{-}\right)=I_{i k}\left(x_{i}\left(t_{k}\right)\right), \quad & i=1,2,
\end{aligned}\right.
$$

where

$$
\begin{aligned}
\left(a_{i}\right)_{2 \times 1} & =\left(\begin{array}{c}
1.6+(2 / \pi) \arctan |u| \\
1.5+(2 / \pi) \arctan |u|
\end{array}\right), \quad\left(b_{i}\right)_{2 \times 1}=\frac{1}{220 \pi}\left(\begin{array}{l}
u \\
u
\end{array}\right), \\
\left(f_{j}\right)_{2 \times 1} & =\left(g_{j}\right)_{2 \times 1}=\frac{1}{2}\left(\begin{array}{c}
\sin u \\
\sin u
\end{array}\right), \\
\left(c_{i j}\right)_{2 \times 2} & =\frac{1}{2640 \pi}\left(\begin{array}{cc}
\sin 2 t & \cos 2 t \\
\cos 2 t & \sin 2 t
\end{array}\right), \\
\left(d_{i j}\right)_{2 \times 2} & =\frac{1}{2640 \pi}\left(\begin{array}{cc}
\sin ^{2} t & \cos ^{2} t \\
\cos ^{2} t & \sin ^{2} t
\end{array}\right),
\end{aligned}
$$




$$
\begin{array}{cl}
\left(e_{i}\right)_{2 \times 1}= & \frac{\pi}{2}\left(\begin{array}{c}
\sin t \\
\cos t
\end{array}\right), \quad\left(\tau_{i j}\right)_{2 \times 2}=\left(\begin{array}{ll}
1 & 1 \\
1 & 1
\end{array}\right), \\
\left(I_{i k}\right)_{2 \times 2}= & \frac{1}{240}\left(\begin{array}{cc}
-u & -u \\
-u & -u
\end{array}\right), \\
& \omega=2 \pi, \quad[0,2 \pi]_{\mathbb{T}} \cap\left\{t_{k}: k \in \mathbb{N}\right\}=\left\{t_{1}, t_{2}\right\} .
\end{array}
$$

When $\mathbb{T}=\mathbb{R}$ or $\mathbb{Z}$, system (5.1) has at least one exponentially stable $\pi$-antiperiodic solution.

Proof. By calculation, we have $a_{1}^{m}=1.6, a_{2}^{m}=1.5, a_{1}^{M}=2.6, a_{2}^{M}=2.5$, $\nu_{1}=\nu_{2}=2 / \pi, l_{1}=0.6-1 / \pi, l_{2}=0.5-1 / \pi, \rho_{1}=\rho_{2}=1 /(220 \pi), \delta_{1}=\delta_{2}=$ $1 /(220 \pi), L_{1}=L_{2}=1 / 2, P_{1}=P_{2}=1 / 2, f_{1}^{M}=f_{2}^{M}=1 / 2, g_{1}^{M}=g_{2}^{M}=1 / 2$, $c_{11}^{L}=c_{12}^{L}=c_{21}^{L}=c_{22}^{L}=1 /(2640 \pi), d_{11}^{L}=d_{12}^{L}=d_{21}^{L}=d_{22}^{L}=1 /(2640 \pi)$, $e_{1}^{L}=e_{2}^{L}=\pi / 2, G_{11}=G_{12}=G_{21}=G_{22}=1 / 240$.

It is obvious that $\left(\mathrm{H}_{1}\right)-\left(\mathrm{H}_{5}\right),\left(\mathrm{H}_{7}\right)-\left(\mathrm{H}_{9}\right)$ and $\left(\mathrm{H}_{11}\right)$ are satisfied. Furthermore, we can easily calculate that

$$
\Pi=\left(h_{i j}\right)_{2 \times 2} \approx\left(\begin{array}{cc}
0.1926 & -0.4396 \\
-0.4392 & 0.0978
\end{array}\right)
$$

is a nonsingular $M$ matrix, thus $\left(\mathrm{H}_{6}\right)$ is satisfied.

Case 1. When $\mathbb{T}=\mathbb{R}$, then $\mu(t)=0$. Take $\varepsilon=0.01$, we have that

$$
\max _{t \in[0, \omega / 2]_{\mathbb{T}}} \Theta_{1}(\varepsilon, t) \approx-0.2697<0 \text { and } \max _{t \in[0, \omega / 2]_{\mathbb{T}}} \Theta_{2}(\varepsilon, t) \approx-0.1697<0 .
$$

Hence $\left(\mathrm{H}_{10}\right)$ holds. By Theorems 3.1 and 4.1, system (5.1) has at least one exponentially stable $\pi$-anti-periodic solution.

Case 2. When $\mathbb{T}=\mathbb{Z}$, then $\mu(t)=1$. Take $\varepsilon=0.001$, we have that

$$
\max _{t \in[0, \omega / 2]_{\mathbb{T}}} \Theta_{1}(\varepsilon, t) \approx-0.1233<0 \text { and } \max _{t \in[0, \omega / 2]_{\mathbb{T}}} \Theta_{2}(\varepsilon, t) \approx-0.1135<0 .
$$

Hence $\left(\mathrm{H}_{10}\right)$ holds. By Theorems 3.1 and 4.1, system (5.1) has at least one exponentially stable $\pi$-anti-periodic solution.

\section{REFERENCES}

[1] A.R. Aftabizadeh, S. Aizicovici and N.H. Pavel, On a class of second-order antiperiodic boundary value problems, J. Math. Anal. Appl. 171 (1992), 301-320.

[2] R. Agarwal, M. Bohner and A. Peterson, Inequalities on times cales: a survey, Math. Inequality Appl. 4 (4) (2001), 535-557.

[3] C. BAI, Stability analysis of Cohen-Grossberg BAM neural networks with delays and impulses, Chaos Solitons Fractals 35 (2008), 263-267.

[4] M. Bohner, M. FAN AND J. ZhANG, Existence of periodic solutions in predator-prey and competition dynamic systems, Nonlinear Anal. Real World Appl. 7 (2006), 1193-1204.

[5] M. Bohner And A. Peterson, Dynamic Equations on Time Scales, an Introduction with Applications, Boston, Birkhäuser, 2001.

[6] H.L. Chen, Antiperiodic wavelets, J. Comput. Math. 14 (1996), 32-39. 
[7] Y. Chen, J.J. Nieto and D. O'Regan, Anti-periodic solutions for fully nonlinear firstorder differential equations, Math. Comput. Modelling 46 (2007), 1183-1190.

[8] Y.Q. Chen, Anti-periodic solutions for semilinear evolution equations, J. Math. Anal. Appl. 315 (2006), 337-348.

[9] Z. Chen, D. ZhaO AND X. Fu, Discrete analogue of high-order periodic Cohen-Grossberg neural networks with delay, Appl. Math. Comput. 214 (2009), 210-217.

[10] M.A. Cohen And S. Grossberg, Stability and global pattern formulation and memory storage by competitive neural networks, IEEE Trans. Syst. Man Cybern. 13 (1983), 815826.

[11] F.J. Delvos And L. Knoche, Lacunary interpolation by antiperiodic trigonometric polynomials, BIT 39 (1999), 439-450.

[12] J.Y. Du, H.L. HAN AND G.X. Jin, On trigonometric and paratrigonometric Hermite interpolation, J. Approx. Theory 131 (2004), 74-99.

[13] G. Guseinov, Integration on time scales, J. Math. Anal. Appl. 285 (2003), 107-127.

[14] J.J. HopfiEld, Neurons with graded response have collective computational properties like those of two-state neurons, Proc. Natl. Acad. Sci. 81 (1984), 3088-3092.

[15] E.R. Kaufmann And Y.N. RAFfoul, Periodic solutions for a neutral nonlinear dynamical equation on a time scale, J. Math. Anal. Appl. 319 (2006), 315-325.

[16] V. Lakshmikantham and, A.S. Vatsala, Hybird systems on time scales, J. Comput. Appl. Math. 141 (2002), 227-235.

[17] T. Li AND S. FeI, Stability analysis of Cohen-Grossberg neural networks with time-varying and distributed delays, Neurocomputing 71 (2008), 1069-1081.

[18] T. LI, S. FeI, Y. Guo AND Q. ZHU, Stability analysis on Cohen-Grossberg neural networks with both time-varying and continuously distributed delays, Nonlinear Anal. Real World Appl. 10 (2009), 2600-2612.

[19] Y.K. LI, Existence and stability of periodic solutions for Cohen-Grossberg neural networks with multiple delays, Chaos Solitons Fractals 20 (2004), 459-466.

[20] Y.K. Li, X.R. Chen And L. ZhaO, Stability and existence of periodic solutions to delayed Cohen-Grossberg BAM neural networks with impulses on time scales, Neurocomputing 72 (2009), 1621-1630.

[21] Y.K. Li AND X. FAN, Existence and globally exponential stability of almost periodic solution for Cohen-Grossberg BAM neural networks with variable coefficients, Appl. Math. Modelling 33 (2009), 2114-2120.

[22] Y.K. Li AND L. YAng, Anti-periodic solutions for Cohen-Grossberg neural networks with bounded and unbounded delays, Commun. Nonlinear Sci. Numer. Simulat. 14 (2009), 31343140 .

[23] X.F. LiaO, C.D. Li and K. Wong, Criteria for exponential stability of Cohen-Grossberg neural networks, Neural Networks 17 (2004), 1401-1414.

[24] J.L. MAwhin, Topological degree methods in nonlinear boundary value problems, CBMS Regional Conference Series in Mathematics, vol. 40, Amer. Math. Soc., Providence, RI, 1979.

[25] Y. Meng, L. Huang and Z. Yuan, Exponential stability analysis of Cohen-Grossberg neural networks with time-varying delays, Acta Math. Appl. Sin. English Ser. 28 (2012), $181-192$.

[26] D. O'Regan, Y.J. Cho and Y.Q. Chen, Topological Degree Theory and Application, Taylor \& Francis Group, Boca Raton, London, New York, 2006.

[27] H. Окосні, On the existence of periodic solutions to nonlinear abstract equations, J. Math. Soc. Japan 40 (1988), 541-553. 
[28] _ On the existence of anti-periodic solutions to nonlinear parabolic equations in noncylindrical domains, Nonlinear Anal. 14 (1990), 771-783.

[29] J.Y. SHAO, Anti-periodic solutions for shunting inhibitory cellular neural networks with time-varying delays, Phys. Lett. A 372 (2008), 5011-5016.

[30] R. Wu, An anti-periodic LaSalle oscillation theorem, Appl. Math. Lett. 21 (2008), 928933.

[31] Z. YANG AND D. XU, Impulsive effects on stability of Cohen-Grossberg neural networks with variable delays, Appl. Math. Comput. 177 (2006), 63-78.

[32] Z.C. YANG AND D.Y. XU, Impulsive effects on stability of Cohen-Grossberg neural networks with variable delays, Appl. Math. Comput. 177 (2006), 63-78.

[33] Y. YIN, Monotone iterative technique and quasilinearization for some anti-periodic problems, Nonlinear World 3 (2) (1996), 253-266.

Manuscript received March 30, 2012

Yongkun Li AND Tianwei Zhang

Department of Mathematics

Yunnan University

Kunming, Yunnan 650091, P.R. CHINA

E-mail address: yklie@ynu.edu.cn, zhang@kmust.edu.cn 